\title{
Mudanças ocorridas após a cirurgia bariátrica: revisão integrativa da literatura
}

\author{
Changes that occurred after bariatric surgery: an integrative literature review \\ Cambios después de la cirugía bariátrica: revisión integradora de la literatura
}

Recebido: 25/02/2021 | Revisado: 07/03/2021 | Aceito: 09/03/2021 | Publicado: 17/03/2021

Aline Zulin
ORCID: https://orcid.org/0000-0001-6749-762X
Universidade Estadual de Maringá, Brasil
E-mail: aline_zulin@ hotmail.com
Thamires Fernandes Cardoso da Silva Rodrigues
ORCID: http://orcid.org/0000-0001-7942-4989
Universidade Estadual de Maringá, Brasil
E-mail: tfcsrodrigues@gmail.com
Luana Cristina Bellini Cardoso
ORCID: https://orcid.org/0000-0001-8460-1177
Universidade Estadual de Maringá, Brasil
E-mail: luana.bellini @ hotmail.com
Fernanda Gatez Trevisan dos Santos
ORCID: https://orcid.org/0000-0001-7082-6949
Universidade Estadual de Maringá, Brasil
E-mail: fer.gatez@gmail.com
Anderson da Silva Rêgo
ORCID: https://orcid.org/0000-0002-0988-5728
Universidade Estadual de Maringá, Brasil
E-mail: andersondsre@gmail.com
Luiza Djegov Franco de Oliveira
ORCID: https://orcid.org/0000-0001-7811-0427
Universidade Paulista, Brasil
E-mail: djegov@gmail.com
Cremilde Aparecida Trindade Radovanovic
ORCID: https://orcid.org/0000-0001-9825-3062
Universidade Estadual de Maringá, Brasil
E-mail: kikanovic2010@ hotmail.com

\section{Resumo}

Objetivo: identificar as evidências disponíveis na literatura sobre as alterações que ocorreram na vida de pessoas após a cirurgia bariátrica. Métodos: revisão integrativa da literatura, com busca de estudos publicados entre janeiro de 2016 a fevereiro de 2020, realizada nas bases de dados: Cochrane Library, Embase (Elsevier), US National Library of Medicine (Pubmed) e Biblioteca Virtual em Saúde (BVS) no mês de abril de 2020. As buscas foram obtidas pelo cruzamento, nas bases de dados, dos seguintes descritores: Obesity, Bariatric Surgery, Quality of life. Para a organização dos resultados foi utilizado o fluxograma preconizado pelo PRISMA. Para a operacionalização dos resultados, adotou-se o software Iramuteq ${ }^{\circledR}$. Resultados: 14 artigos compuseram esta revisão, os quais apresentaram as principais mudanças após a realização da cirurgia bariátrica. Os achados foram repartidos em três classes: Transformações físicas após a cirurgia bariátrica; Melhora na qualidade de vida após a cirurgia bariátrica; e Estigma associado ao procedimento cirúrgico como estratégia para a perda de peso. Conclusão: identificou-se que as mudanças na vida das pessoas após a cirurgia bariátrica perpassam aspectos físicos, psicoemocionais e nos relacionamentos interpessoais.

Palavras-chave: Obesidade; Cirurgia bariátrica; Qualidade de vida.

\begin{abstract}
Objective: to identify the evidence available in the literature about the changes that occurred in the lives of people after bariatric surgery. Methods: integrative literature review, with a search for studies published between January 2016 and February 2020, carried out in the following databases: Cochrane Library, Embase (Elsevier), US National Library of Medicine (Pubmed) and Virtual Health Library (VHL) in April 2020. Searches were obtained by crossing, in the databases, the following descriptors: Obesity, Bariatric Surgery, Quality of life. For the organization of the results, the flowchart recommended by PRISMA was used. For the operationalization of the results, the Iramuteq ${ }^{\circledR}$ software was adopted. Results: 14 articles comprised this review, which presented the main changes after performing bariatric surgery. The findings were divided into three classes: Physical changes after bariatric surgery; Improvement in quality of life after bariatric surgery; and Stigma associated with the surgical procedure as a strategy for weight
\end{abstract}


loss. Conclusion: it was identified that the changes in people's lives after bariatric surgery pervade physical, psychoemotional and interpersonal relationships.

Keywords: Obesity; Bariatric surgery; Quality of life.

\section{Resumen}

Objetivo: identificar la evidencia disponible en la literatura sobre los cambios ocurridos en la vida de las personas después de la cirugía bariátrica. Métodos: revisión integradora de la literatura, con búsqueda de estudios publicados entre enero de 2016 y febrero de 2020, realizada en las bases de datos: Cochrane Library, Embase (Elsevier), US National Library of Medicine (Pubmed) y Virtual Health Library (VHL) en Abril de 2020. Las búsquedas se obtuvieron cruzando, en las bases de datos, los siguientes descriptores: Obesidad, Cirugía bariátrica, Calidad de vida. Para la organización de los resultados se utilizó el diagrama de flujo recomendado por PRISMA. Para la operacionalización de los resultados se adoptó el software Iramuteq ${ }^{\circledR}$. Resultados: 14 artículos conformaron esta revisión, que presentaron los principales cambios tras la realización de la cirugía bariátrica. Los hallazgos se dividieron en tres clases: cambios físicos después de la cirugía bariátrica; Mejora de la calidad de vida después de la cirugía bariátrica; y estigma asociado al procedimiento quirúrgico como estrategia para adelgazar. Conclusión: se identificó que los cambios en la vida de las personas luego de la cirugía bariátrica invaden las relaciones físicas, psicoemocionales e interpersonales.

Palavras clave: Obesidad; Cirugía bariátrica; Calidad de vida.

\section{Introdução}

A cirurgia bariátrica tem se tornado crescente em pessoas que têm necessidade de reduzir grande quantidade de peso e minimizar os efeitos das comorbidades oriundas a obesidade. No mundo, em 2017, mais de quatro milhões de pessoas morreram por causas relacionadas ao excesso de peso (World Health Organization, 2021). Nesse sentido, define-se obesidade como o acúmulo anormal ou excessivo de gordura que apresenta risco à saúde, considerando-se um índice de massa corporal (IMC) acima de 30 (World Health Organization, 2021).

A obesidade consiste em um problema de relevância para a saúde pública, que envolve processos biopsicossociais, cujos encargos negativos afetam a saúde e a qualidade de vida das pessoas (Dias et al., 2017). A qualidade de vida pode ser definida como a percepção do indivíduo a respeito da posição que ocupa na vida, no contexto cultural, no sistema de valores e em relação aos objetivos, padrões e preocupações que possui (World Health Organization Quality of Life Assessment, 1995). As dificuldades enfrentadas pelas pessoas com obesidade perpassam por atividades cotidianas, como comprar roupas, utilizar os meios de transporte, adentrar no mercado de trabalho e manter relações socioafetivas (Oliveira et al., 2018).

Assim, a obesidade associa-se à maior probabilidade de dificuldade de interação social, baixa autoestima, estresse, depressão e isolamento social (Gordon, Kaio \& Sallet, 2011). Estudos demonstraram que a taxa de indivíduos com autopercepção negativa da imagem corporal era mais comum em sujeitos com obesidade (Hamurcu et al., 2015; Sánchez, Dijkstra \& Visser, 2015). Tais aspectos representam, em parte, os principais motivos pela busca por cirurgia bariátrica (Oliveira et al., 2018).

Diante deste contexto, a Organização Mundial da Saúde (OMS) classifica a obesidade como uma das dez doenças mais perigosas, devendo ser tratada de forma eficaz, de modo que a cirurgia bariátrica pode ser uma alternativa para a perda de peso mais duradoura (World Health Organization, 2017). Assim, torna-se importante, esta revisão de literatura, investigar se a perda de peso secundária a cirurgia bariátrica influencia na melhora da qualidade de vida. Nessa perspectiva, esse estudo teve como objetivo identificar as evidências disponíveis na literatura sobre as alterações que ocorreram na vida da pessoa após a cirurgia bariátrica.

\section{Metodologia}

Trata-se de uma revisão integrativa da literatura, ferramenta que permite sumarizar resultados provenientes de pesquisas sobre um tema, de forma sistemática, ordenada e abrangente (Ercole, Melo \& Alcoforado, 2014) A realização da 
pesquisa bibliográfica auxilia para aprofundar no tema e somar conhecimentos. A pesquisa e a leitura norteiam o pesquisador a se aperfeiçoar, conhecendo mais o tema e desenvolvendo habilidades e competências cognitivas (Pereira et al., 2018).

Para iniciar a coleta, formulou-se a seguinte pergunta de pesquisa: Quais evidências existentes na literatura sobre as mudanças na vida de pessoas com obesidade após a cirurgia bariátrica? A coleta ocorreu no mês de abril de 2020, consultando as seguintes bases de dados: Cochrane Library, Embase (Elsevier), US National Library of Medicine (Pubmed) e Biblioteca Virtual em Saúde (BVS). Para realizar a busca, utilizou-se a combinação de palavras-chave extraídas dos Descritores em Ciências da Saúde (DeCS) e Medical Subject Headings (Mesh), associadas por meio do operador booleano "AND": Obesity AND Bariatric Surgery AND Quality of life.

Os critérios de inclusão adotados foram: artigos primários, publicados no período de janeiro de 2016 a fevereiro de 2020, disponíveis na íntegra eletronicamente, período pós-operatório de seis meses a dois anos, não se restringiu quanto ao idioma e localidade de origem das produções. Os critérios de exclusão foram: participantes reoperados, grupo controle com participantes não operados, participantes não operados, não relatar o período de pós-operatório, editoriais, revisão de literatura, teses/dissertações e resumos.

A seleção dos estudos ocorreu de modo simultâneo por dois pesquisadores, as discordâncias foram examinadas até que houvesse consenso. Os artigos duplicados nas bases foram excluídos após a leitura na íntegra para evitar erros de exclusões. Para colher os dados dos estudos, elaborou-se uma planilha no Microsoft Excel com as seguintes informações: identificação, título do estudo, ano de publicação, país de origem, periódico, objetivo do estudo, método, resultados, conclusão e nível de evidência.

Para o nível de evidência, considerou-se a seguinte classificação: nível I - revisões sistemáticas ou metanalises de Ensaios Clínicos Randomizados Controlados (ECRC); nível II - evidências obtidas de pelo menos um ECRC bem delineado; nível III - ensaios clínicos bem delineados sem randomização; nível IV - provenientes de estudo de caso controle e de coorte bem delineados; nível V - obtidos de revisões sistemáticas de estudos descritivos ou qualitativos; nível VI - oriundos de um único estudo descritivo ou qualitativo; e nível VII - evidências originárias de opinião de autoridades e/ou comitê de especialistas (Galvão, 2006).

Para a operacionalização dos resultados, adotou-se o software Iramuteq ${ }^{\circledR}$ (acrônimo de $\mathrm{R}$ pour les Analyses Multidimensionnelles de Textes et de Questionnaires) 0.7 ALFA 2.3.3.1, que a partir da confecção de um corpus textual contendo os desfechos e as conclusões dos autores dos artigos selecionados, pode-se identificar a frequência dos vocábulos que deram origem aos segmentos de textos (equivale a aproximadamente 3,25 linhas). Adotou-se a Classificação Hierárquica Descendente (CHD), cujos segmentos de texto foram classificados conforme a associação entre os vocábulos semelhantes e, então, flexionados em função da frequência, formando classes iniciais (Souza et al., 2018). Inicialmente o software indicou a participação de sete classes, a partir da convergência entre os resultados e a literatura, organizou-se em três classes finais, nomeadas como: Classe I - Transformações físicas após a cirurgia bariátrica; Classe II - Melhora na qualidade de vida após a cirurgia bariátrica; e Classe III - Estigma associado ao procedimento cirúrgico como estratégia para a perda de peso.

Realizou-se a síntese qualitativa dos estudos. Ressalta-se que, por tratar-se de uma revisão integrativa, cujas informações são de domínio público, dispensou-se apreciação ética, todas as recomendações contidas na Resolução nº 466/2012 foram respeitadas.

\section{Resultados}

Compuseram a amostra dessa revisão, 14 artigos. O processo de busca e seleção dos estudos é demonstrado no fluxograma preconizado pelo PRISMA representado na Figura 1. 
Figura 1. Fluxograma do processo de seleção dos estudos para a revisão integrativa, 2020.

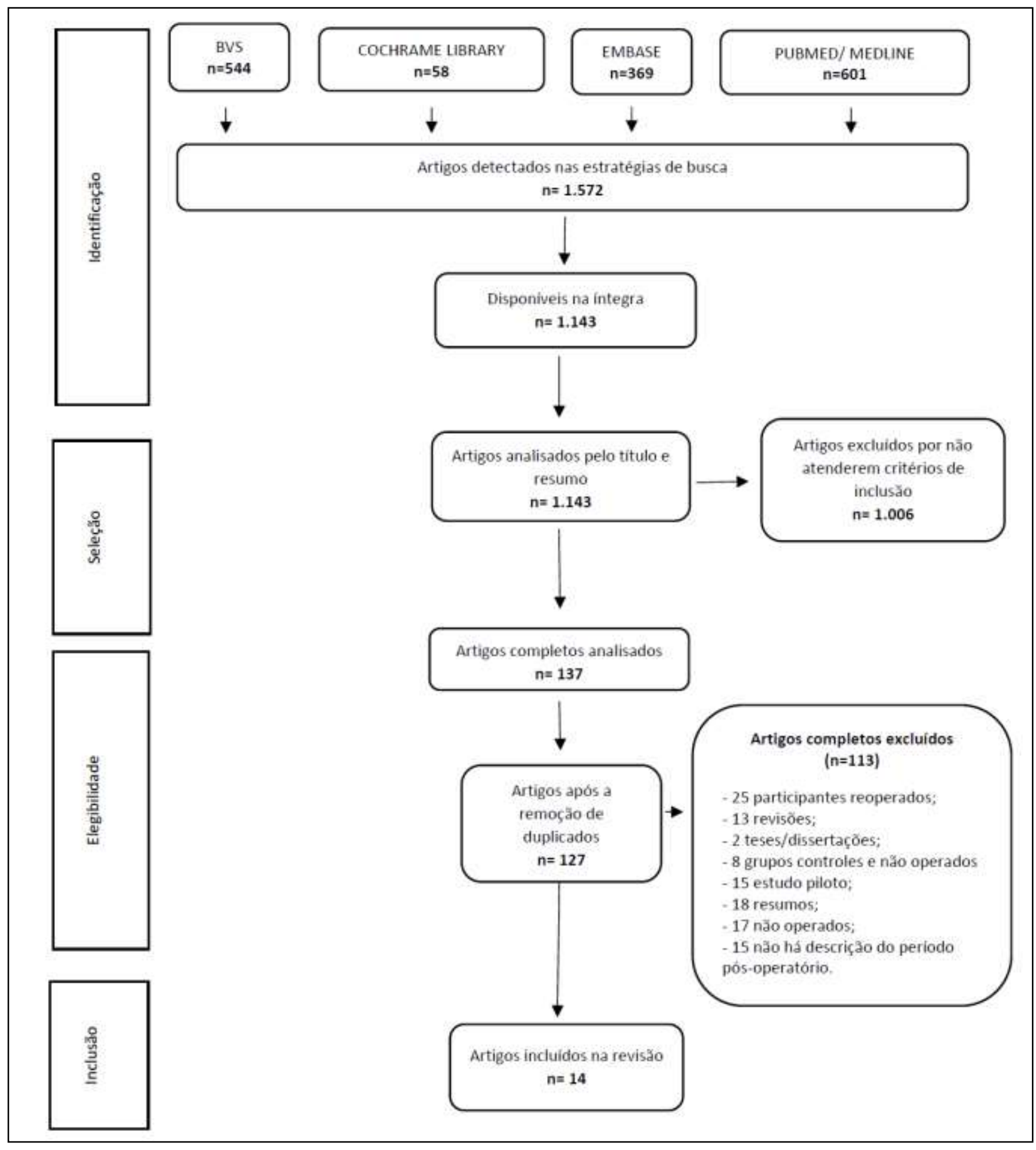

Fonte: As autoras (2021), Moher et al. (2009).

Todos os estudos foram publicados na língua inglesa e do país de origem. As obras selecionadas foram em sua maioria quantitativas, os estudos de natureza qualitativa adotaram como referencial metodológico a teoria fundamentada nos dados (Griauzde et al., 2018; Graham et al., 2017) e a análise temática (Edward et al., 2018). A população dos estudos variou de seis a 304 participantes (média de 94 participantes), com idade entre 18 e 70 anos (média de 42 anos). A caracterização dos resultados está descrita no Quadro 1. 
Quadro 1. Caracterização dos artigos selecionados.

\begin{tabular}{|c|c|c|c|c|c|}
\hline ID & Título & $\begin{array}{c}\text { País/ } \\
\text { periódico }\end{array}$ & $\begin{array}{c}\text { Objetivo/Desenho } \\
\text { metodológico }\end{array}$ & Desfecho & $\begin{array}{c}\text { Nível de } \\
\text { Evidência }\end{array}$ \\
\hline $\begin{array}{l}\text { Oliveira et } \\
\text { al., } 2018\end{array}$ & $\begin{array}{l}\text { Bariatric surgery } \\
\text { repercussions on the } \\
\text { quality of life, } \\
\text { biochemical profile, } \\
\text { and blood pressure of } \\
\text { patients with morbid } \\
\text { obesity }\end{array}$ & $\begin{array}{l}\text { Brasil/ } \\
\text { Fisioterapia e } \\
\text { Pesquisa }\end{array}$ & $\begin{array}{l}\text { Analisar as repercussões da } \\
\text { cirurgia bariátrica na } \\
\text { qualidade de vida, no perfil } \\
\text { bioquímico e na pressão } \\
\text { arterial de indivíduos obesos } \\
\text { mórbidos em três momentos } \\
\text { distintos: um mês antes, três } \\
\text { meses depois e seis meses } \\
\text { após a cirurgia bariátrica. } \\
\text { Estudo descritivo, longitudinal } \\
\text { e prospectivo. }\end{array}$ & $\begin{array}{l}\text { A cirurgia bariátrica pode } \\
\text { repercutir positivamente na } \\
\text { qualidade de vida, na melhora do } \\
\text { perfil bioquímico e na pressão } \\
\text { arterial de participantes obesos } \\
\text { após três e seis meses a cirurgia. }\end{array}$ & II \\
\hline $\begin{array}{c}\text { Lechaux, } \\
\text { Foll \& } \\
\text { Rascle, } 2020\end{array}$ & $\begin{array}{l}\text { Weight Loss and } \\
\text { Quality of Life After } \\
\text { Sleeve Gastrectomy } \\
\text { or One-Anastomosis } \\
\text { Gastric Bypass: } \\
\text { Results of a } \\
\text { Prospective Study of } \\
120 \text { Women with } \\
\text { Morbid Obesity }\end{array}$ & $\begin{array}{l}\text { França/ } \\
\text { Obesity } \\
\text { Surgery }\end{array}$ & $\begin{array}{l}\text { Comparar a qualidade de vida } \\
\text { após a gastrectomia vertical } \\
\text { laparoscópica (SG) e bypass } \\
\text { gástrico laparoscópico com } \\
\text { uma anastomose em } \\
\text { participantes adultas do sexo } \\
\text { feminino obesas nos primeiros } \\
\text { dois anos após a cirurgia. } \\
\text { Estudo prospectivo. }\end{array}$ & $\begin{array}{l}\text { As técnicas de gastrectomia } \\
\text { vertical laparoscópica e bypass } \\
\text { gástrico laparoscópico com uma } \\
\text { anastomose são eficazes na } \\
\text { redução do índice de massa } \\
\text { corporal e na melhoria da } \\
\text { qualidade de vida. }\end{array}$ & IV \\
\hline $\begin{array}{l}\text { Akkayaoğlu } \\
\text { \& Celik, } \\
2020\end{array}$ & $\begin{array}{l}\text { Eating attitudes, } \\
\text { perceptions of body } \\
\text { image and patient } \\
\text { quality of life before } \\
\text { and after bariatric } \\
\text { surgery }\end{array}$ & $\begin{array}{l}\text { Turquia/ } \\
\text { Applied } \\
\text { Nursing } \\
\text { Research }\end{array}$ & $\begin{array}{l}\text { Examinar atitudes alimentares, } \\
\text { percepções da imagem } \\
\text { corporal e qualidade de vida } \\
\text { de participantes antes e após a } \\
\text { cirurgia bariátrica. } \\
\begin{array}{l}\text { Estudo prospectivo e } \\
\text { descritivo. }\end{array}\end{array}$ & $\begin{array}{l}\text { A avaliação multidimensional dos } \\
\text { participantes e a implementação } \\
\text { das intervenções de enfermagem } \\
\text { contribuíram positivamente na vida } \\
\text { de indivíduos submetidos à cirurgia } \\
\text { bariátrica. }\end{array}$ & VI \\
\hline $\begin{array}{l}\text { Yilmaz \& } \\
\text { Yilmaz, } \\
2019\end{array}$ & $\begin{array}{l}\text { Effect on Quality of } \\
\text { Life of the Changing } \\
\text { Body Image of } \\
\text { Individuals with } \\
\text { Obesity Surgery }\end{array}$ & $\begin{array}{l}\text { Turquia/ } \\
\text { Bariatric } \\
\text { Surgical } \\
\text { Practice and } \\
\text { Patient Care }\end{array}$ & $\begin{array}{l}\text { Avaliar os efeitos da alteração } \\
\text { da imagem corporal e } \\
\text { qualidade de vida de } \\
\text { indivíduos que se submeteram } \\
\text { a cirurgia bariátrica. Estudo } \\
\text { prospectivo. }\end{array}$ & $\begin{array}{l}\text { A cirurgia bariátrica alterou } \\
\text { positivamente a imagem corporal } \\
\text { dos indivíduos e a qualidade de } \\
\text { vida. }\end{array}$ & VI \\
\hline $\begin{array}{l}\text { Cherick et } \\
\text { al., } 2019\end{array}$ & $\begin{array}{lr}\text { Bariatric } & \text { Surgery } \\
\text { Significantly } & \\
\text { Improves the Quality } \\
\text { of Sexual Life and } \\
\text { Self-esteem } \\
\text { Morbidly in } \\
\text { Women }\end{array}$ & $\begin{array}{l}\text { Itália/ } \\
\text { Obesity } \\
\text { Surgery }\end{array}$ & $\begin{array}{l}\text { Analisar a função sexual, os } \\
\text { sintomas depressivos e a } \\
\text { autoestima de mulheres com } \\
\text { obesidade mórbida submetidas } \\
\text { à cirurgia bariátrica. } \\
\text { Estudo prospectivo. }\end{array}$ & $\begin{array}{l}\text { A cirurgia bariátrica resulta em } \\
\text { uma melhora significativa na } \\
\text { qualidade de vida sexual, sintomas } \\
\text { depressivos e autoestima nas } \\
\text { mulheres com obesidade mórbida. }\end{array}$ & VI \\
\hline $\begin{array}{l}\text { Castanha et } \\
\text { al., } 2018\end{array}$ & $\begin{array}{l}\text { Avaliação } \\
\text { qualidade de vida, } \\
\text { perda de peso e } \\
\text { comorbidades de } \\
\text { participantess } \\
\text { submetidos à cirurgia } \\
\text { bariátrica }\end{array}$ & $\begin{array}{l}\text { Brasil/ } \\
\text { Revista do } \\
\text { Colégio } \\
\text { Brasileiro de } \\
\text { Cirurgiões }\end{array}$ & $\begin{array}{l}\text { Mensurar a eficácia da perda } \\
\text { de peso, analisar a evolução de } \\
\text { comorbidades, investigar a } \\
\text { qualidade de vida e avaliar o } \\
\text { protocolo BAROS (Bariatric } \\
\text { Analysis and Reporting } \\
\text { Outcome System) no pós- } \\
\text { operatório de participantes } \\
\text { submetidos à cirurgia } \\
\text { bariátrica. } \\
\text { Estudo transversal e } \\
\text { quantitativo. }\end{array}$ & $\begin{array}{l}\text { A cirurgia bariátrica demonstrou } \\
\text { ser eficaz no tratamento da } \\
\text { obesidade mórbida e no controle } \\
\text { das comorbidades. A qualidade de } \\
\text { vida foi avaliada de forma positiva } \\
\text { através do protocolo BAROS. }\end{array}$ & VI \\
\hline $\begin{array}{l}\text { Major et al., } \\
2016\end{array}$ & $\begin{array}{l}\text { The Impact of } \\
\text { Preoperative Body } \\
\text { Weight on Quality of } \\
\text { Life after Surgical } \\
\text { Treatment for Morbid } \\
\text { Obesity }\end{array}$ & $\begin{array}{l}\text { Polônia/ } \\
\text { Bariatric } \\
\text { Surgical } \\
\text { Practice and } \\
\text { Patient Care }\end{array}$ & $\begin{array}{l}\text { Analisar } \text { o efeito } \\
\text { tratamento } \text { do } \\
\text { obesidade com gastrectomia } \\
\text { vertical laparoscópica ou } \\
\text { bypass gástrico laparoscópico } \\
\text { na qualidade de vida dos } \\
\text { participantes tratados. Estudo } \\
\text { prospectivo. }\end{array}$ & $\begin{array}{l}\text { O tratamento cirúrgico resultou em } \\
\text { melhora significativa em todos os } \\
\text { parâmetros de qualidade de vida. } \\
\text { Não foi encontrada relação } \\
\text { significativa entre a melhora da } \\
\text { qualidade de vida e o índice de } \\
\text { massa corporal inicial ou o tipo de } \\
\text { cirurgia. }\end{array}$ & VI \\
\hline Vegel et al., & Patient-reported & Estados & Identificar preditores de uma & Participantes de cirurgia bariátrica & VI \\
\hline
\end{tabular}




\begin{tabular}{|c|c|c|c|c|c|}
\hline 2017 & $\begin{array}{l}\text { quality of life after } \\
\text { bariatric } \\
\text { single } \\
\text { analysis. }\end{array}$ & $\begin{array}{l}\text { Unidos da } \\
\text { América/ } \\
\text { Journal of } \\
\text { Surgical } \\
\text { Research }\end{array}$ & $\begin{array}{l}\text { maior qualidade de vida } \\
\text { relatada pelos participantes no } \\
\text { pós-operatório. } \\
\text { Estudo qualitativo. }\end{array}$ & $\begin{array}{l}\text { experimentaram melhora } \\
\text { significativa na qualidade de vida } \\
\text { um ano após cirurgia. }\end{array}$ & \\
\hline $\begin{array}{l}\text { Tarride et } \\
\text { al., } 2017\end{array}$ & 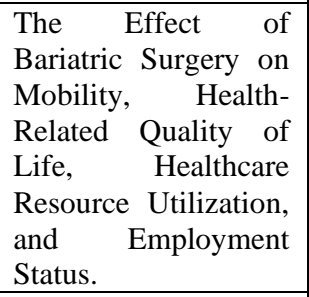 & $\begin{array}{l}\text { Canadá/ } \\
\text { Obesity } \\
\text { Surgery }\end{array}$ & $\begin{array}{l}\text { Avaliar o impacto da cirurgia } \\
\text { bariátrica sobre mobilidade e } \\
\text { situação laboral. Estudo } \\
\text { prospectivo. }\end{array}$ & $\begin{array}{l}\text { A cirurgia bariátrica tem um } \\
\text { grande impacto na mobilidade e } \\
\text { qualidade de vida. }\end{array}$ & VI \\
\hline $\begin{array}{l}\text { Alkassis et } \\
\text { al., } 2019\end{array}$ & \begin{tabular}{lr}
\multicolumn{2}{l}{ Quality of Life before } \\
and after & Sleeve \\
Gastrectomy & in \\
Lebanese Population.
\end{tabular} & $\begin{array}{l}\text { Líbano/ } \\
\text { Journal } \\
\text { Obesity }\end{array}$ & $\begin{array}{l}\text { Determinar o impacto da } \\
\text { cirurgia bariátrica nas } \\
\text { comorbidades associadas à } \\
\text { obesidade. } \\
\text { Estudo prospectivo. } \\
\end{array}$ & $\begin{array}{l}\text { A gastrectomia vertical melhora a } \\
\text { qualidade de vida e permite a } \\
\text { redução de comorbidades. }\end{array}$ & IV \\
\hline $\begin{array}{l}\text { Griauzde et } \\
\text { al., } 2018\end{array}$ & $\begin{array}{l}\text { Understanding the } \\
\text { psychosocial impact } \\
\text { of weight loss } \\
\text { following bariatric } \\
\text { surgery: a qualitative } \\
\text { study. }\end{array}$ & $\begin{array}{l}\text { Estados } \\
\text { Unidos/ } \\
\text { BMC } \\
\text { Obesity }\end{array}$ & $\begin{array}{l}\text { Entender melhor as } \\
\text { experiências dos participantes } \\
\text { após a cirurgia bariátrica. } \\
\text { Estudo qualitativo. }\end{array}$ & $\begin{array}{l}\text { A perda de peso após a cirurgia } \\
\text { bariátrica leva as mudanças } \\
\text { complexas na auto percepção e na } \\
\text { relação } \\
\text { relacionamentos, que podem ser } \\
\text { mediadores proximais de } \\
\text { resultados de saúde mental } \\
\text { comumente avaliados, como } \\
\text { depressão. }\end{array}$ & VI \\
\hline $\begin{array}{c}\text { Graham et } \\
\text { al., } 2017\end{array}$ & $\begin{array}{l}\text { Patient experiences } \\
\text { of adjusting to life in } \\
\text { the first } 2 \text { years after } \\
\text { bariatric surgery: a } \\
\text { qualitative study. }\end{array}$ & $\begin{array}{l}\text { Reino Unido/ } \\
\text { Clinical } \\
\text { Obesity }\end{array}$ & $\begin{array}{l}\text { Explorar como as pessoas se } \\
\text { ajustam aos aspectos sociais } \\
\text { de suas vidas nestes primeiros } \\
\text { dois anos após a cirurgia. } \\
\text { Estudo qualitativo. }\end{array}$ & $\begin{array}{l}\text { Os participantes relutavam em } \\
\text { discutir suas experiências de } \\
\text { cirurgia em situações sociais. } \\
\text { Dentro dos ambientes sociais, as } \\
\text { discussões em torno da cirurgia } \\
\text { bariátrica foram relatadas como } \\
\text { uma fonte de preocupação com } \\
\text { relação aos riscos potenciais de } \\
\text { revelar ter feito a cirurgia bariátrica } \\
\text { por ser julgado por outras pessoas, } \\
\text { o que pode afetar negativamente a } \\
\text { adaptação à vida após a cirurgia. }\end{array}$ & VI \\
\hline $\begin{array}{l}\text { Mendes } \\
\& \text { Vargas, } \\
2017\end{array}$ & $\begin{array}{l}\text { Quality of life after } \\
\text { vertical gastrectomy } \\
\text { evaluated by the } \\
\text { baros questionnaire }\end{array}$ & $\begin{array}{l}\text { Brasil/ } \\
\text { ABCD } \\
\text { Arquivo } \\
\text { Brasileiro de } \\
\text { Cirurgia } \\
\text { Digestiva }\end{array}$ & 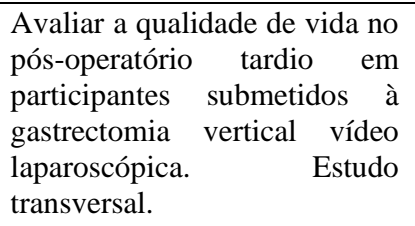 & $\begin{array}{l}\text { A perda de peso foi fundamental } \\
\text { para melhorar a qualidade de vida e } \\
\text { ofereceu a resolução ou melhora } \\
\text { clínica em todos as comorbidades } \\
\text { investigadas em participantes } \\
\text { submetidos à gastrectomia vertical. }\end{array}$ & VI \\
\hline $\begin{array}{c}\text { Edward et } \\
\text { al., } 2018\end{array}$ & \begin{tabular}{lr}
\multicolumn{2}{l}{ Personal Descriptions } \\
of Life & Before and \\
After & Bariatric \\
Surgery & From \\
Overweight & or Obese \\
Men. &
\end{tabular} & $\begin{array}{l}\text { Austrália/ } \\
\text { American } \\
\text { Journal of } \\
\text { Men's Health }\end{array}$ & $\begin{array}{l}\text { Explorar descrições de } \\
\text { participantes bariátricos do } \\
\text { sexo masculino antes e depois } \\
\text { cirurgia e, especificamente, } \\
\text { sua adaptação a uma nova vida } \\
\text { estilo, novos limites após a } \\
\text { cirurgia e iluminar quaisquer } \\
\text { barreiras potenciais para } \\
\text { procurar consulta para } \\
\text { bariátrico cirurgia. } \\
\text { Estudo qualitativo, descritivo } \\
\text { exploratório. }\end{array}$ & $\begin{array}{l}\text { A experiência masculina de } \\
\text { adaptação à cirurgia pós-bariátrica, } \\
\text { imagem corporal, e as mudanças } \\
\text { exploradas neste estudo } \\
\text { identificaram áreas que podem ser } \\
\text { direcionadas para melhorar } \\
\text { resultados de médio a longo prazo } \\
\text { após a cirurgia bariátrica. }\end{array}$ & VI \\
\hline
\end{tabular}

Fonte: Autores (2021).

Dos artigos, notou-se que a técnica cirúrgica de Sleeve e Bypass gástrico, foram os procedimentos mais frequentes (Lechaux, Foll \& Rascle, 2020; Akkayaoglu \& Çelik, 2020; Cherick et al., 2019; Castanha et al., 2018; Major et al., 2016; Alkassis et al., 2019; Griauzde et al., 2018; Mendes \&Vargas, 2017; Vegel et al., 2017; Tarride et al., 2016). Somente um 
estudo (Edward et al., 2018) foi realizado exclusivamente com participantes do sexo masculino. O estudo de Graham et al (2018) demonstrou a percepção de participantes e a sua adaptação após a cirurgia bariátrica, tais dados podem ser utilizados por profissionais de saúde, a fim de assisti-los no período pré e pós-operatório.

Com base nos achados e na organização dos dados realizada por meio do software IRAMUTEQ ${ }^{\circledR}$, cujo corpus textual resultante foi repartido em 52 segmentos de texto, deste 45 foram analisados ( $87 \%$ do total), dividiu-se os resultados em três classes finais, denominadas: Classe I - Transformações físicas após a cirurgia bariátrica; Classe II - Melhora na qualidade de vida após a cirurgia bariátrica; e Classe III - Estigma associado ao procedimento cirúrgico como estratégia para a perda de peso, conforme Figura 2.

Figura 2. Dendograma das classes. Paraná, Brasil, 2021.

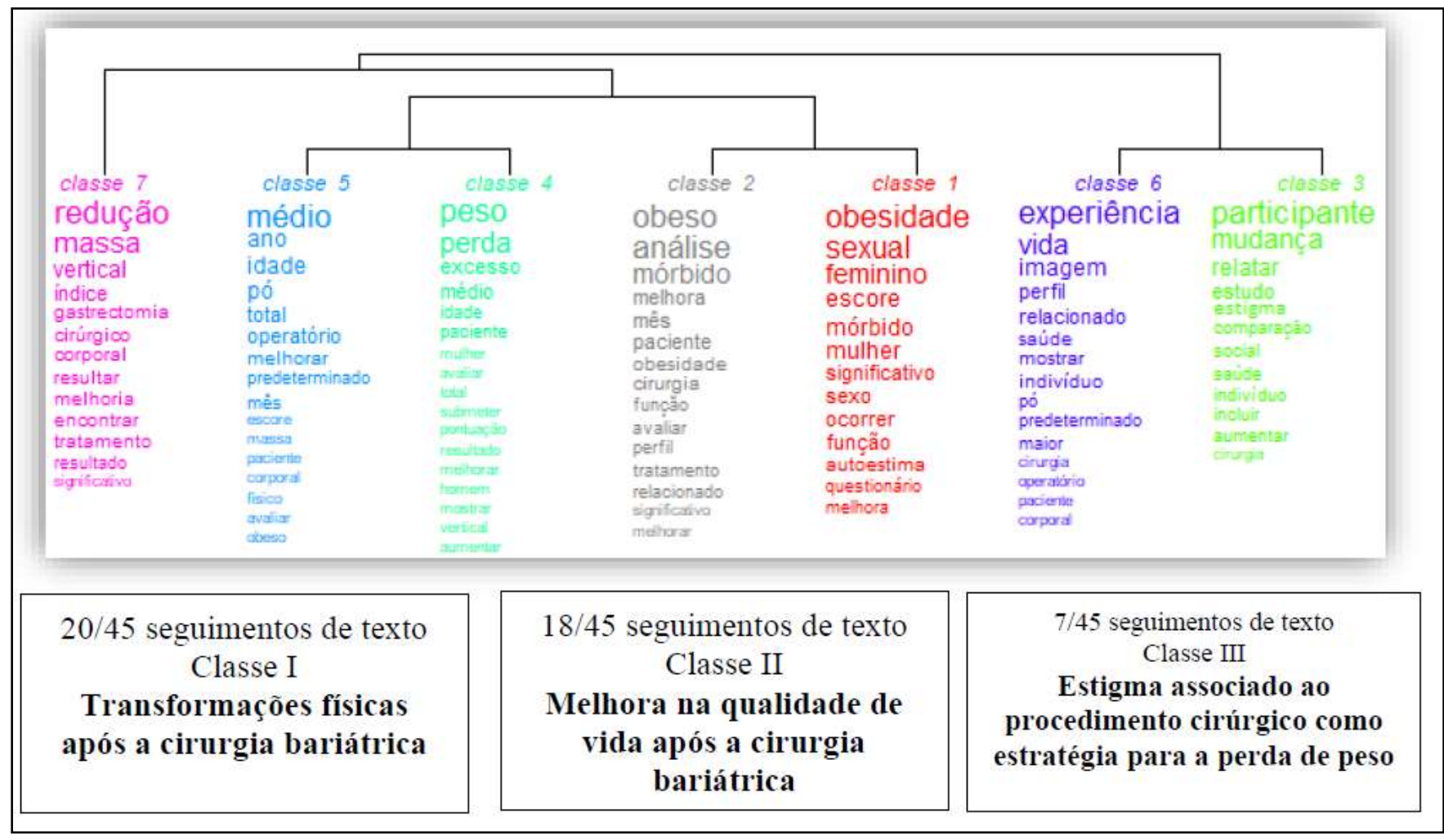

Fonte: Dados organizados no software IRAMUTEQ ${ }^{\circledR}(2021)$.

\section{Classe I. Transformações físicas após a cirurgia bariátrica}

Nesta classe, notou-se que os participantes apresentaram redução média no peso de 42,07\% após a cirurgia bariátrica. Ao diminuir o peso corporal, houve melhora no perfil bioquímico (Oliveira et al., 2018; Castanha et al., 2018; Alkassis et al., 2019; Mendes \& Vargas, 2017). Observou-se também, que a cirurgia bariátrica foi eficaz para minimizar os efeitos e/ou sintomas das comorbidades, como diabetes mellitus e hipertensão arterial (Lechaux, Foll \& Rascle, 2020; Yilmaz \& Yilmaz, 2019; Castanha et al., 2018; Vegel et al., 2017; Alkassis et al., 2019; Mendes \& Vargas, 2017).

\section{Classe II. Melhora na qualidade de vida após a cirurgia bariátrica}

$\mathrm{Na}$ classe II, demonstrou-se que a perda de peso impactou positivamente a qualidade de vida dos participantes que se submeteram a cirurgia bariátrica (Oliveira et al., 2018; Lechaux, Foll \& Rascle, 2020; Yilmaz \& Yilmaz, 2019; Major et al., 2016; Vegel et al., 2017; Alkassis et al., 2019; Mendes \& Vargas, 2017; Tarride et al., 2017), observando-se aumento na satisfação em relação ao corpo, melhora na função sexual, nos sintomas depressivos, na autoestima e nas relações interpessoais 
(Cherick et al., 2019; Griauzde et al., 2018). Além destes, após a cirurgia bariátrica, os participantes modificaram seus hábitos de vida, adotando uma alimentação saudável e tornando-se mais ativos (Akkayaoğlu \& Çelik, 2020), o que resultou na redução de dores e desconfortos associadas a atividades do cotidiano (Tarride et al., 2017).

\section{Classe III. Estigma associado ao procedimento cirúrgico como estratégia para a perda de peso}

$\mathrm{Na}$ terceira classe notou-se que, apesar de os participantes relatarem os desafios vivenciados pela obesidade e que após a cirurgia bariátrica houve transformações positivas em suas vidas, nos aspectos biopsicossociais (Edward et al., 2018). Estudos demonstraram que alguns participantes evitaram expor o método utilizado para a perda de peso ou a intenção de se submeter ao procedimento, devido ao receio de sofrer com os julgamentos e o estigma social implicado a cirurgia bariátrica pelos amigos e/ou familiares (Graham et al., 2017; Edward et al., 2018).

\section{Discussão}

A partir desta revisão integrativa, pode-se notar que as principais implicações associadas à cirurgia bariátrica para a vida dos participantes foram: redução do IMC: a melhora do perfil bioquímico, qualidade de vida, relacionamentos interpessoais e mudança nos hábitos de vida. A cirurgia bariátrica trata-se de uma estratégia útil para o tratamento da obesidade grave, que auxilia na resolução de comorbidades (Hood et al., 2016; Medina, Jiménez \& Sanchez, 2018).

Pesquisa demonstrou que pessoas com obesidade grave possuem seis vezes mais chances de desenvolver disfunção pulmonar em relação aos demais indivíduos, e à medida que ocorre a redução de peso aumenta-se o volume pulmonar (Pereira et al., 2018). Identificou-se que a cirurgia bariátrica foi eficaz para promover aumento na capacidade cardiorrespiratória, minimizar a frequência dos distúrbios pulmonares e progresso nas variáveis antropométricas (Lizama et al., 2017; Pereira et al., 2018).

Os achados desta revisão corroboram estudo desenvolvido no Paraná, cuja perda de peso através da cirurgia bariátrica mostrou-se eficaz para a redução dos valores séricos dos lipídios e potencial redução do risco cardiometabólico (Rêgo et al., 2020). Resultados semelhantes foram obtidos em Minas Gerais, visto que após o procedimento cirúrgico houve melhora do perfil bioquímico e na pressão arterial de participantes (Oliveira et al., 2018).

Nesse aspecto, a redução de peso através da cirurgia bariátrica, promove a diminuição do IMC e a longo prazo a melhora na qualidade de vida das pessoas com obesidade (Hofstadt et al., 2017), os quais começam a ser percebidos dois anos do procedimento (Gryth, 2019). Assim, após a cirurgia bariátrica há mudanças significativas no cotidiano das pessoas, no âmbito físico, emocional, profissional e social. A melhora é percebida, em especial, na qualidade de vida após o procedimento (Eduardo et al., 2017; Mendes \& Vargas, 2017; Kirkil et al., 2018). A qualidade de vida advém, em parte, pela diminuição da dor e de limitações físicas, com consequente aumento na mobilidade (Campbell et al., 2018; Fermont et al., 2017).

Não obstante, a redução de peso modifica a imagem corporal, facilitando as relações conjugais e interpessoais (Yilmaz \& Yilmaz, 2019). A libido e as questões sexuais são um incômodo para quem convive com a obesidade. Entretanto, após a cirurgia bariátrica, as pessoas relatam ter mais autoconfiança, maior prazer e satisfação na vida sexual (Vegel et al., 2018; Twells et al., 2017). Observou-se ainda, a redução dos sintomas depressivos e melhoria do bem-estar geral (Efferdinger et al., 2017; Lizama et al., 2017). Assim a perda de peso após a cirurgia bariátrica suscita mudanças complexas na auto percepção e nos relacionamentos interpessoais, que podem ser mediadores proximais de resultados de saúde mental como a depressão (Griauzde et al., 2018). Os resultados desta revisão apontam que as transformações positivas na vida dos participantes associaram-se a cirurgia e a modificação nos hábitos de vida. Estudo desenvolvido com candidatos a cirurgia bariátrica que foram acompanhados por educadores físicos, identificou mudança nos hábitos de vida com consequente redução na percepção de dor e na melhora da qualidade de vida (Barreto et al., 2018). 
Apesar de os benefícios relatados após a cirurgia bariátrica, detectou-se que alguns participantes demonstraram receio em expor o método adotado para a perda de peso. Estudo demonstra que a sociedade estigmatiza pessoas com obesidade e aquelas que escolher perder peso por meio de procedimentos cirúrgicos (Hübner et al., 2016).

O estigma relacionado ao peso apresenta consequências negativas para o bem-estar físico e psicológico dos indivíduos (Nolann \& Eshleman, 2016; Pearl et al., 2017). A exposição do indivíduo com obesidade ativa ao estigma de peso onde experimentar esse estigma é estressante para quem se percebe obeso, independente do IMC, apresentando estresse, o cortisol aumenta e com isso a pessoa tem mais vontade de se alimentar, contribuindo para o aumento de peso (Himmelstein et al., 2015).

Em pesquisa realizada com estudantes universitários observou-se que os mesmos apresentavam estigma em relação as pessoas com obesidade devido a imagem corporal, revelando ser um problema social, prevalente e prejudicial. Resgatando a realidade de aumentar a compreensão das pessoas que o excesso de peso se tornou um vício em alimentos e alterações do comportamento alimentar, onde pessoas com obesidade precisam de ajuda e não de estigmas sociais (O’Brien et al., 2020). A dependência alimentar pode trazer atitudes estigmatizantes para o próprio indivíduo obeso, ser rotulado como dependente de alimento pode ser prejudicial ao bem-estar psicológico e prejudicar a pessoa nas tentativas de perda de peso (Ruddock et al., 2019).

Os efeitos negativos do estigma de peso em pessoas com obesidade são referentes a avaliação social negativa à sua aparência, trazendo aumento do afeto negativo e a aparência da autoestima, atribuindo sinais de preconceito social podendo levar a problemas físicos e psicológicos (Gerend et al., 2020).

Dentre as limitações dessa revisão, destaca-se a escassez de ensaios clínicos, cujos resultados poderiam fornecer maior rigor aos efeitos da cirurgia bariátrica na vida das pessoas. Outro ponto consiste no fato de não ter contemplado estudos com maior tempo de acompanhamento, o que forneceria um panorama, a longo prazo, sobre as implicações do procedimento cirúrgico. Entretanto, os resultados demonstram a importância do acompanhamento interdisciplinar dos sujeitos submetidos a cirurgia bariátrica, por um período mínimo de um ano após o procedimento, para que as transformações físicas, psicoemocionais e interpessoais oriundas possam ser vivenciadas de modo saudável.

\section{Conclusão}

Identificou-se a partir dessa revisão, que após a cirurgia bariátrica pessoas com obesidade passam por transformações em suas vidas, que perpassam aspectos físicos, psicoemocionais e nos relacionamentos interpessoais. Grande parte das mudanças se relacionaram a perda de peso sustentada, a redução dos agravos oriundos as comorbidades, melhoria do perfil bioquímico, da qualidade de vida, interação social, conjugal e aumento na autoestima. Apesar destes benefícios, alguns participantes dos estudos relataram receio em declarar os meios utilizados para a perda de peso, visto o estigma associado ao procedimento.

Sugere-se para estudos futuros entender, através de pesquisas qualitativas, as mudanças ocorridas após a cirurgia bariátrica, se essas mudanças foram significativas para a vida no seu contexto físico, social e psicoemocional e se a perda de peso se mantém sustentada para essas mudanças permanecerem em suas vidas.

\section{Agradecimentos}

O presente trabalho foi realizado com apoio da Coordenação de Aperfeiçoamento de Pessoal de Nível Superior Brasil (CAPES) - Código de Financiamento 001. 


\section{Referências}

Akkayaoğlu, H., \& Çelik, S. (2020). Eating attitudes, perceptions of body image and patient quality of life before and after bariatric surgery. Applied Nursing Research, 53, 151270. https://doi.org/10.1016/j.apnr.2020.151270

Alkassis, M., Haddad, F. G., Gharios, J., Noun, R., \& Chakhtoura, G. (2019). Quality of Life before and after Sleeve Gastrectomy in Lebanese Population. Hindawi Journal of Obesity, 2019, 1952538. https://doi.org/10.1155/2019/1952538

Barreto, B. L. M., Lima, J. S., Albuquerque, D. B., Kreimer, F., Ferraz, A. A. B., \& Campos, J. M. (2018). Atividade física, qualidade de vida e imagem corporal de participantess candidatos a cirurgia bariátrica. ABCD Arq Bras Cir Dig, 31(1), e1349. http://dx.doi.org/10.1590/0102-672020180001e1349.

Campbell, J. A., Hensher, M., Neil, A., Alison Venn, A., Stephen Wilkinson, S., \& Palmer, A. J. (2018). An Exploratory Study of Long-Term Publicly Waitlisted Bariatric Surgery Patients' Quality of Life Before and 1 Year After Bariatric Surgery, and Considerations for Healthcare Planners. Pharmaco Economics Open, 2(1), 63-76. https://doi.org/10.1007/s41669-017-0038-z

Castanha, C. R., Ferraz, A. A. B., Castanha, A. R., Belo, G. Q. M. B., Lacerda, R. M. R., Vilar, L. (2018). Avaliação da qualidade de vida, perda de peso e comorbidades de participantes submetidos à cirurgia bariátrica. Rev Col Bras Cir, 45(3), e1864. https://doi.org/ 10.1590/0100-6991e-20181864.

Cherick, F., Te, V., Anty, R., Turchi, L., Benoit, M., Schiavo, L., \& Iannelli, A. (2019). Bariatric Surgery Significantly Improves the Quality of Sexual Life and Self-esteem in Morbidly Obese Women. Obesity Surgery, 29(5), 1576-1582. https://doi.org/10.1007/s11695-019-03733-7

Cofre-Lizama, A., Delgado-Floody, P., Angulo-Díaz, P., \& Jerez-Mayorga, D. (2017). Intervención integral de ocho meses disminuye el peso y mejora los niveles de depresión y ansiedad en obesos severos y mórbidos. Rev Fac Med, 65(2), 239-43. http://dx.doi.org/10.15446/revfacmed.v65n2.59595

Dias, P. C., Henriques, P., Anjos, L. A., \& Burlandy, L. (2017). Obesidade e políticas públicas: concepções e estratégias adotadas pelo governo brasileiro. Cad Saúde Pública, 33(7), e00006016. https://doi.org/10.1590/0102-311x00006016.

Edward, K. L., Hii, M. W., Giandinoto, J-A., Hennessy, J., \& Thompson, L. (2018). Personal descriptions of life before and after bariatric surgery from overweight or obese men. Am J Mens Health, 12(2), 265-273. https://doi.org/10.1177/1557988316630770

Eduardo, C. A., Silva, A. A., Cruz, C. D., Viana, G. C. P., \& Rocha, R. L. P. (2017). Cirurgia bariátrica: a percepção dos participantes frente ao impacto físico, psicológico e social. RECOM, 7, e1173. https://doi.org/10.19175/recom.v7i0.1173

Efferdinger, C., König, D., Klaus, A., \& Jagsch, R. (2017). Emotion regulation and mental well-being before and six months after bariatric surgery. Eat Weight Disord, 22(2), 353-360. https://doi.org/10.1007/s40519-017-0379-8

Ercole, F. F., Melo, L. S., \& Alcoforado, C. L. G. C. (2014). Integrative review versus systematic review. Rev Min Enferm, 18(1), 1-260. https://dx.doi.org/10.5935/1415-2762.20140001. 2014.

Fermont, J. M., Blazeby, J. M., Rogers, C. A., \& Wordsworth, S. (2017). The EQ-5D-5L is a valid approach to measure health related quality of life in patients undergoing bariatric surgery. PLoS One, 12(12), e0189190. https://doi.org/10.1371/journal.pone.0189190

Franssens, B. T., Hoogduin, H., Leiner, T., Graaf, Y. V-D., \& Visseren, F. L. J. (2017). Relation between brown adipose tissue and measures of obesity and metabolic dysfunction in patients with cardiovascular disease. J Magn Reson Imaging, 46(2), 497-504. https://doi.org/10.1002/jmri.25594

Galvão, C. M. (2006). Níveis de evidência. Acta Paul. Enferm, 19(2). http://dx.doi.org/10.1590/S0103-21002006000200001

Gerend, M. A., Sutin, A. R., Terracciano, A., \& Maner, J. K. (2020). The role of psychological attribution in responses to weight stigma. Obes Sci Pract, 6(5), 473-483. https://doi.org/10.1002/osp4.437

Gordon, P. C., Kaio, G. H., \& Sallet, P. C. (2011). Aspectos do acompanhamento psiquiátrico de participantes obesos sob tratamento bariátrico: revisão. Rev Psiquiatr Clin, 38(4), 148-54. http://dx.doi.org/10.1590/S0101-60832011000400007

Graham, Y., Hayes, C., Small, P. K., Mahawar, K., \& Ling, J. (2017). Patient experiences of adjusting to life in the first 2 years after bariatric surgery: a qualitative study. Clinical Obesity, 7(5), 323-335. https://doi.org/10.1111/cob.12205

Griauzde, D. H., Ibrahim, A. M., Fisher, N., Stricklen, A., Ross, R., \& Ghaferi, A. A. (2018). Understanding the psychosocial impact of weight loss following bariatric surgery: a qualitative study. BMC Obes, 5(38). https://doi.org/10.1186/s40608-018-0215-3

Gryth, K., Persson, C., Näslund, I., Sundbom, M., Näslund, E., \& Stenberg, E. (2019). The Influence of Socioeconomic Factors on Quality-of-Life After Laparoscopic Gastric Bypass Surgery. Obesity Surgery, 29(11), 3569-3576. https://doi.org/10.1007/s11695-019-04028-7

Hamurcu, P., Öner, C., Telatar, B., \& Yeșildağ, S. (2015). Obezitenin benlik saygısı ve beden algısı uzerine etkisi. Türk Aile Hek Derg, 19(3), 122-129. $10.15511 /$ tahd.15.02122

Himmelstein, M. S., Belsky, A. C. I., \& Tomiyama, A. J. (2015). The Weight of Stigma: Cortisol Reactivity to Manipulated Weight Stigma. Obesity, 23(2), 368-374. https://doi.org/10.1002/oby.20959

van der Hofstadt, C. J., Cubas, S. E., Tirado-González, S., Pérez-Martínez, E., Sebastián, S. O., Caballero, J. L. E., Rodríguez-Marín, J., \& Leal-Costa, C. (2017). Evolución de la calidad de vida a los 24 meses de seguimiento em participantes sometidos a cirugía bariátrica: comparación entre el by-pass gástrico y la gastrectomía vertical tubular. An Sist Sanit Navar, 40(2), 199-209. http://dx.doi.org/10.23938/assn.0032

Hood, M. M., Corsica, J., Bradley, L., Wilson, R., Chirinos, D. A., \& Vivo, A. (2016). Managing severe obesity: understanding and improving treatment adherence in bariatric surgery. J Behav Med, 39(6), 1092-1103. https://doi.org/10.1007/s10865-016-9772-4 
Hübner, C., Schmidt, R., Selle, J., Köhler, H., Müller, A., Zwaan, M., \& Hilbert, A. (2016). Comparing Self-Report Measures of Internalized Weight Stigma: The Weight Self-Stigma Questionnaire versus the Weight Bias Internalization Scale. PLoS One, 11(10), e0165566. https://doi.org/10.1371/journal.pone.0165566

Kirkil, C., Aygen, E., Korkmaz, M. F., \& Bozan, M. B. Quality of life after vertical gastrectomy evaluated by the baros questionnaire. ABCD Arq Bras Cir Dig, 31(3), e1385. https://doi.org/10.1590/0102-672020180001e1385

Kolotkin, R. L., Smolarz, B. G., Meincke, H. H., \& Fujioka, K. (2018). Improvements in health-related quality of life over 3 years with liraglutide 3.0mg compared with placebo in participants with overweight or obesity. Clinical Obesity, 8(1), 1-10. https://doi.org/10.1111/cob.12226

Lakatos, E. M., \& Marconi, M. A (2017). Metodologia do Trabalho Científico. 8. ed. São Paulo: Atlas.

Lechaux, D., Foll, D. L., \& Rascle, O. (2020). Weight Loss and Quality of Life After Sleeve Gastrectomy or One-Anastomosis Gastric Bypass: Results of a Prospective Study of 120 Women with Morbid Obesity. Obes Surg, 30(7), 2828-2831. https://doi.org/10.1007/s11695-020-04442-2

Major, P., Matłok, M., Pędziwiatr, M., Migaczewski, M., Budzyński, P., Stanek, M., Kisielewski, M., Natkaniec, M., \& Budzyński, A. (2015). Quality of life after bariatric surgery. Obes Surg, 25(9), 1703-1710. https://doi.org/10.1007/s11695-015-1601-2

Major, P., Michał Pędziwiatr, M., Dworak, J., Pisarska, M., Lasek, A., Wierdak, M., Natkaniec, M., \& Budzyński, A. (2016). The Impact of Preoperative Body Weight on Quality of Life after Surgical Treatment for Morbid Obesity. Bariatric Surg Practice and Patient Care, 11(4). https://doi.org/10.1089/bari.2016.0020

Medina, R., Jiménez, H. C., \& Sánchez, L. C. (2018). Comportamiento de la pérdida de peso en los participantess intervenidos con cirugía bariátrica en un hospital de tercer nível, 2013-2016. Rev Colomb Cir, 33(3), 257-64. http://dx.doi.org/10.30944/20117582.70

Mendes, G. A., \& Vargas, G. P. (2017). Qualidade de vida após gastrectomia vertical avaliada pelo questionário BAROS. ABCD Arq Bras Cir Dig, 30(1), 1417. http://dx.doi.org/10.1590/0102-6720201700010005

Moher, D., Liberati, A., Tetzlaff, J., \& Altman, D. G. (2009). The PRISMA Group. Preferred reporting items for systematic reviews and meta analyses: the PRISMA statement. PLoS Med, 6(7), e1000097. http://dx.doi.org/10.1371/journal.pmed1000097.

Nolan, L. J., \& Eshleman, A. (2016). Paved with good intentions: Paradoxical eating responses to weight stigma. Appetite, 102, 15-24. https://doi.org/10.1016/j.appet.2016.01.027

O`Brien, K. S., Puhl, R. M., Latner, J. D., Lynott, D., Reid, J. D., Vakhitova, Z., \& Hunter, J. A. (2020). The effect of a food addiction explanation model for weight control and obesity on weight stigma. Nutrients, 12(2), 294. https://doi.org/10.3390/nu12020294

Oliveira, L. S. F., Mazini Filho, M. L., Castro, L. B. P., Touguinha, H. M., Silva, P. C. R., \& Ferreira, M. E. C. (2018). Bariatric surgery repercussions on the quality of life, biochemical profile, and blood pressure of patients with morbid obesity. Fisioter Pesqui, 25(3), 284-293. http://dx.doi.org/10.1590/18092950/17017725032018

Pearl, R. L., Wadden, T. A., Hopkins, C. M., Shaw, J. A., Hayes, M. R., Bakizada, Z. M., \& Alamuddin, N. (2017). Association between weight bias internalization and metabolic syndrome among treatment-seeking individuals with obesity. Obesity, 25(2), 317-322. https://doi.org/10.1002/oby.21716

Pereira, L. M. C., Aidar, F. J., Matos, D. G., Farias Neto, J. P., Souza, R. F., Sousa, A. C. S., ... \& Silva Júnior, W. M (2019). Assessment of Cardiometabolic Risk Factors, Physical Activity Levels, and Quality of Life in Stratified Groups up to 10 Years after Bariatric Surgery. Int J Environ Res Public Health, 16(11), 1975. https://doi.org/10.3390/ijerph16111975

Pereira, R. B., Rivera, I. R., Brito, Z. C. T., \& Silva, M. A. M. (2018). Efeito da obesidade na fisiologia e distúrbio pulmonar antes e após a cirurgia bariátrica. Cienc Cuid Saude, 17(1). https://doi.org/10.4025/cienccuidsaude.v17i1.34368

Pereira, A. S., Shitsuka, D. M., Parreira, F. J., \& Shitsuka, R. (2018). Metodologia da pesquisa científica. 1. ed. Santa Maria: UFSM, NTE. [E-book].

Rêgo, A. S., Zulin, A., Scolari, S., Cardoso, L. C. B., Sanches, R. C. N., Salci, M. A., \& Radovanovic, C. A. T. (2020). Effect of sleeve gastrectomy on lipid parameters and cardiometabolic risk in young women. Rev Col Bras Cir, 47, e253. https://doi.org/10.1590/0100-6991e-20202537

Ruddock, H. K., Orwin, M., Boyland, E. J., Evans, E. H., \& Hardman, C. A. (2019). Obesity Stigma: Is the 'Food Addiction' Label Feeding the Problem? Nutrients, 11(9), 2100. https://doi.org/10.3390/nu11092100

Sanchez, C. M., Dijkstra, S. C., \& Visser, M. (2015). Self-perception of body weight status in older Dutch adults. J Nutr Health Aging, 19, 612-618. https://doi.org/10.1007/s12603-015-0486-Z

Souza, M. A. R., Wall, M. L., Thuler, A. C. M. C., Lowen, I. M. V., \& Peres, A. M. (2018). The use of IRAMUTEQ software for data analysis in qualitative research. Rev Esc Enferm USP, 52, e03353. http://dx.doi.org/10.1590/S1980-220X2017015003353

Tarride, J. E., Breau, R., Sharma, A. M., Hong, D., Gmora, S., Guertin, J. R., \& O'Reilly, D., (2017). The Effect of Bariatric Surgery on Mobility, HealthRelated Quality of Life, Healthcare Resource Utilization, and Employment Status. Obes Surg, 27(2), 349-356. https://doi.org/10.1007/s11695-016-2298-6

Twells, L. K., Driscoll, S., Gregory, D. M., Lester, K., Fardy, J. M., \& Pace, D. (2017). Morbidity and health-related quality of life of patients accessing laparoscopic sleeve gastrectomy: a single-centre cross-sectional study in one province of Canada. BMC Obesity, 4, 40. https://doi.org/10.1186/s40608-017$0176-\mathrm{y}$

The WHOQOL group (1995). The World Health Organization quality of life assessment (WHOQOL): position paper from the World Health Organization. Soc Sci Med, 41(10). https://doi.org/10.1016/0277-9536(95)00112-K

Vegel, A. J., Shah, N., Lidor, A. O., Greenberg, J. A., Shan, Y., Wang, X., \& Funk, L. M. (2017). Patient-reported quality of life after bariatric surgery: a single institution analysis. J Surg Res, 218, 117-123. https://doi.org/10.1016/j.jss.2017.05.068 
Research, Society and Development, v. 10, n. 3, e31410313329, 2021

(CC BY 4.0) | ISSN 2525-3409 | DOI: http://dx.doi.org/10.33448/rsd-v10i3.13329

Yilmaz, P., \& Yilmaz, M (2019). Effect on Quality of Life of the Changing Body Image of Individuals with Obesity Surgery. Bariatric Surg Practice and Patient Care, 14(2). https://doi.org/10.1089/bari.2018.0034

World Health Organization (2021). Obesity [internet]. WHO; 2021. https://www.who.int/health-topics/obesity\#tab=tab_2.

World Health Organization (2017). Obesity and overweight [internet]. WHO; 2021. http://www.who.int/mediacentre/factsheets/fs311/en/ 